\title{
How to Be Humean about Idealization Laws
}

\author{
Toby Friend (D)
}

Department of Philosophy, University of Bristol, Bristol, UK

Email: toby.friend@bristol.ac.uk

(Received 26 February 2021; revised 22 October 2021; accepted 21 February 2022; first published online 04 March 2022)

\section{Abstract}

If one has Humean inclinations, what account should one provide for idealization laws? I introduce the currently most popular Humean approach to laws of nature, the best systems account, along with some basic requirements for how to be Humean. I then show why idealization laws are unlikely to be accommodated within this account of laws. Finally, I offer an alternative approach that takes idealization laws to be meta-laws, placing requirements on the theorems of the best system.

\section{Introduction and plan}

If one has Humean inclinations, what account should one provide for idealization laws? In section 2, I introduce the currently most popular Humean approach to laws of nature, the best systems account, along with some basic requirements for how to be Humean. In section 3, I show why idealization laws are unlikely to be accommodated within this account. In section 4, I offer an alternative approach that takes idealization laws to be meta-laws, placing requirements on the theorems of a best system.

\section{Some background}

\section{I Grounding laws in the mosaic's regularities}

A Humean mosaic comprises only worldly goings-on that are not intrinsically causal. Maybe it will contain occurrences intrinsically describable as "Charge $x$ is instantiated at spacetime point $p$," or "Mass $x$ is spatiotemporally proximate to mass $y . "$ But it won't contain any occurrences that cannot be intrinsically typed by noncausal, nonmodal language (Lewis 1986, 1994). To be a Humean, one need not believe some or other mosaic is all there is-although this is typical-but one should at least take it as a methodological rule that any empirically motivated claim (e.g., in science or scientifically informed metaphysics) should be, in principle, translatable into terms that commit the claimant to no more worldly entities than those in the mosaic. 
Having taken that on board, Humeans may diverge over how many mosaics there are. The view given to us by Lewis, the progenitor of modern-day Humeanism, is that there is one mosaic per world, and it is carved at the joints of a single set of perfectly objective natural properties. Subsequent Humeans have demurred, either over the objective nature of the properties or their uniqueness (e.g., Loewer 1996; Earman 1986). Cohen and Callender (2009) suggest we oppose both constraints. More specifically, they invite Humeans to consider mosaics that carve the world according to different classes of properties correspondent with the kind terms of different sciences (e.g., thermodynamics, statistical mechanics, fluid dynamics, evolutionary biology). Presumably there are composition relations between the various "tiles" in different mosaics, and we needn't suppose that the composed mosaics (arguably those of the special sciences) have the same coverage as the uncomposed mosaics (arguably those of fundamental physics).

Callander and Cohen's (2010) view has significant appeal because it no longer requires Humeans to be committed to a single and entirely language-independent individuation of the world. It also facilitates informative interrelations among the sciences because there need be no expectation of reduction of the entities in one mosaic to another, and yet we can reasonably explore the compositional dependencies that hold (cf. Schrenk 2017). From here on, I take it that this "science-relativized" view of worlds' mosaics is the preferable picture for Humeans to adopt.

Laws of nature are prominent features of scientific theorizing across the sciences, and any empirically motivated methodology should be able to make sense of that. Consequently, the mosaical grounds Humeans propose for laws of nature will need to satisfy a number of requirements. I'll stress three throughout this discussion. First, the proposed grounds (some or other Humean mosaic) should metaphysically explain, or "ground", the laws. Although we might wonder what exactly metaphysical explanation amounts to, one plausible condition in the case of grounding is that the existence of the grounds should be at least ontologically sufficient for the existence of what is grounded. Second, laws' grounds should render them empirically accessible, such that given those grounds, we can see how it is possible that laws are discovered in a nontrivial manner (Hicks 2018). It cannot be, for instance, that the laws are derived from a priori or logical truths or that their grounds are all so rare and unpredictable that we could never hope to discover any of the laws that are grounded in them. A proposal for laws' grounds need not incorporate an account of the process of laws' discovery, but it should remain consistent with the evident fact that we do come to learn them in a nontrivial way. Third, any proposal about laws' grounds should show why laws have the explanatory relevance they have. It seems evident (and I'll elaborate more on this in section 3.2) that laws have an explanatory relevance beyond the mere epistemic crutches they provide for understanding the world. Something about laws makes them good explainers, regardless of what we happen to know. Despite eschewing notions of laws' governance or fundamentality, even a Humean account of laws' grounds should have something to say about why this is.

I will not say anything more about the nature of mosaics' grounding of laws. It suffices to say that whatever solution is proposed had better satisfy the three considerations just described, namely, metaphysical sufficiency, empirical accessibility, and explanatory power. Obviously, Humeans should also not offer grounds that contradict their own methodological rule. As a consequence, Humeans tend to 
propose regularities in a world's mosaic as the laws' grounds. For although it is coherent to hold on to a regularity-based analysis of laws of nature if one doesn't restrict the regularities to those in keeping with the Humean mosaic (e.g., Tahko 2015; Demarest 2017; Kimpton-Nye 2017, 2021a, 2021b), if one does make such a restriction, then regularities seem to be about the only worldly resource one can go on.

\subsection{Best systems account}

Given that regularities ground the laws, the obvious follow-up question is, which regularities? To answer this, the current trend is a so-called Best Systems Account (BSA), which determines the laws of some mosaic as those generalizations that are theorems in that deductive system that best captures some mosaic's content according to a balance of certain desiderata, including, at the very least, simplicity; comprehensiveness (or "strength"); and, in order to accommodate probabilistic laws, a "fit" with the facts (Lewis 1973, 1983, 1994; Loewer 1996; Schrenk 2007, 2014; Cohen and Callender 2009; Callender and Cohen 2010). ${ }^{1}$ Because the content of the discussion that follows is not so concerned with issues surrounding chance, I will mostly ignore the latter constraint.

The notion of strength in the BSA is invariably cashed out in terms of deducibility of content about a Humean mosaic: laws are theorems of a deductive system, originally, at least, a "deductively closed, axiomatisable set of true sentences" (Lewis 1973, 73). A system is stronger if it entails more information about the mosaic. The strength of a system is to be moderated by its simplicity. A world (arguably, our world) may not be such that the strongest system for any mosaic is achievable without a grotesquely large set of deterministic axioms. If that's the case, considerations of simplicity may demand that some axioms — and hence some laws according to BSA — be indeterministic (Earman 1986, 89). More importantly, some generalizations will be left out altogether, relegated to the "merely accidental."

Admittedly, these considerations prove difficult to provide a precise account for, and one might wonder whether, for instance, considerations of simplicity actually always compete with strength (Roberts 2008; Woodward 2014). But that some balancing acts between them (or perhaps other desiderata as well) are required seems to be agreed upon. Arguably, there must also be some constraint on which generalizations are laws. If it is a theorem that energy is conserved, then it is a theorem that energy is conserved or it's not, but the disjunctive claim is surely not a law. There are various criteria for how this restriction might go (no disjunctions, no quantification over nonnatural classes or properties, etc.). One further option that seems to me both necessary and plausible is to restrict the form of the law. In the quantified sciences, at least, we might propose that laws should have the following schematic form:

$$
(x)(\mathrm{S}(x) \rightarrow \mathcal{F}(x)),
$$

(schema 1)

where $S$ is a "system type" correspondent with the basic properties and kinds of the scientific domain being systematized (e.g., comprising two masses, comprising a

\footnotetext{
${ }^{1}$ Recent defenders of the general Best Systems approach have advocated the inclusion of many more desiderata (e.g. Hicks 2018; Dorst 2019; Jaag and Loew 2020). A discussion of the relevance of these additions goes beyond the scope of this article (though see Friend 2022).
} 
particle in an infinite potential well, comprising predator and prey populations in a habitat), and $\mathcal{F}$ is some functional behavior relating the variable properties of the system. I have defended this formulation of quantitative laws in (Friend 2016) on the grounds that equations alone cannot be interpreted as the full content of laws: they must be conditioned on the class of systems to which the equation is explicitly or implicitly taken to be applicable (see also Hüttemann 2021, §1.1). Adopting the schema provides a plausible restriction on which theorems in a best system are laws, and it seems to accurately capture the content of our most prized quantitative laws, for example, the following:

$$
\begin{gathered}
(x)\left(\text { Two masses }(x) \rightarrow F(x)=G \frac{m_{1}(x) m_{2}(x)}{r^{2}(x)}\right) \\
(x)(\text { Electrical component }(x) \rightarrow \mathrm{J}(x)=\sigma(x) \mathbf{E}(x)) .
\end{gathered}
$$

In what follows, I'll assume schema 1 (or something near enough) is the most transparent form of the laws that appear in a best system. What will remain in question is whether or not idealization laws should fit that schema too.

Implicit in most presentations of the BSA account is that whatever ends up counting as a "best" deductive system will be one that is true. However, Braddon-Mitchell (2001) has suggested that the BSA may be much improved by weighing truth against the other desiderata of strength and simplicity. The BSA renders laws analogous to the algorithms of data compression, and compressions can be greatly improved by permitting some degree of loss of the information compressed. As Braddon-Mitchell emphasizes,

such an account of laws is. .. thoroughly in keeping with the Humean tradition. If, as a Humean, you think that laws are just pervasive regularities in the universe, then you shouldn't be obsessed with exceptionlessness. Surely that is something which, as a conceptual requirement, comes with accounts that involve necessitation. (267)

A BSA that trades truth, in the sense of counting a system's regularities' precision and/or proportion of exceptions, as currency alongside strength and simplicity is therefore perfectly consistent with the Humean idea that laws are grounded in their instances. Such instances would still remain regularities in a mosaic, albeit potentially exception-ridden or imprecise ones.

One key benefit of permitting a trade-off between truth and other desiderata is that it makes it more plausible that "special science" laws of the macroscopic world (which are widely thought to have exceptions) get included within the best system of some mosaic. In particular, the statistical nature of the second law of thermodynamics arguably demands that exception-permitting laws be taken into account (Fenton-Glynn 2016), for it is perfectly consistent with fundamental physical laws that some closed system fails to obey the second law. Because much of the macroscopic world functions only so long as its systems are entropic (i.e., second-law obeying), it is natural to demand that any plausible account of macroscopic laws permit exceptions.

In what follows, I'll assume that the BSA trades in truth, as well as strength, simplicity, and fit. That's not to say, however, that we should tolerate radical 
departures from truth. One point I'll be emphasizing later on, for instance, is that idealization laws themselves appear to be too divergent from the truth to be plausible axioms in even a lossy best system. It remains the case, however, that the Humean need not accept the BSA at all. Nevertheless, my proposal for how the Humean might deal with idealization laws takes the BSA as the point of departure. If the approach turns out to need an overhaul, so will what follows.

\subsection{Satisfying the Humean requirements}

On the face of it, the BSA seems in keeping with the Humean agenda while at the same time looking promising when it comes to the previously mentioned requirements on laws' grounds. Presumably, the local matters of fact that comprise any of the world's mosaics can't be, alone, sufficient to ground a best system (Shumener 2021). What is needed in addition is a totality fact that the sum of local mosaical goings-on exhausts the mosaic of that scientific domain. Of course, totality facts are notorious for having no uncontroversial grounds, but I take it that the Humean engages in no unique problem by relying on them. ${ }^{2}$ In conjunction with such a totality fact, mosaical goings-on are metaphysically sufficient for all the theorems of a best system. Moreover, the mosaical goings-on are exemplary candidates for empirical accessibility. True, some may be far away or require impressive devices to observe, but it is the very foundation of Humean motivation for basing science on mosaical facts that it is superlative in empirical accessibility (in contrast with, e.g., secret connections, powers, nomic necessitation relations, and their ilk).

What of laws' explanatory power? The expectation of most Humeans is that laws' explanatory power comes from unification. Loewer writes that the "L-laws" (i.e., theorems of the BSA),

do explain. They explain by unifying. To say that a regularity is an L-law is to say that it can be derived from the best system of the world. But this entails that it can be unified by connecting it to the other regularities implied by the best system. $(1996,113)$

From this passage alone, one might wonder why the explanatory unifying power of laws is explained by the fact that they themselves are unified. But what BSA theorists like Loewer intend to say is that the unifying structure that confers upon laws their explanatory power is provided by the rules of logical consequence and those propositions from which the whole system is derived: that is, the system's axioms. Hence, Callender and Cohen remark that

recognizing in science the attempt to produce small sets of basic principles as a result of balancing simplicity and informativeness is the central and powerful insight that motivates MRL [read "the BSA"] (and also, we believe, the unificationist theory of explanation). (2009, 3)

\footnotetext{
${ }^{2}$ I'm therefore choosing, as I presume most Humeans will, to reject the first premise of Schumener's (2021) first premise in her argument that Humeanism is false because (i) laws are intrinsic, and (ii) reliance on totality facts makes laws extrinsic.
} 
So, for the BSA theorist, laws explain because they feature in a unifying best system, ultimately entailed by the axioms from which the rest of the system follows. As explainers, therefore, the axioms reign supreme.

To be sure, many have queried if this unification is sufficient to confer adequate explanatory power to the laws. It has been repeatedly objected that Humean laws would be involved in a problematic explanatory circle by virtue of being grounded in the mosaic they are also supposed to explain (Armstrong 1983; Maudlin 2007; Lange 2013, 2018). This is no doubt a serious issue for which there has already been much in the way of response on behalf of the Humean (Loewer 2012; Hicks and van Elswyk 2015; Marshall 2015; Miller 2015; Hicks 2021). I'll mostly bypass this debate, resting my Humean account of idealization laws implicitly on the success of some or other Humean response. However, it's worth making the following two points (which will also be helpful for the account of idealization laws developed later in the article). First, once sold on Humeanism in general, one should generally be content to dispel the need for laws to have any ontic explanatory power over the mosaic about which they generalize. Humeans don't believe there are any such real explanatory relations, and it would not be reasonably immanent to critique the Humeans' specific account of laws for not positing such a relation. Second, it is clear that the theorems of the best system for a mosaic do go beyond the mosaic in some sense because they involve a totality fact that is presumably not part of any mosaic itself - at least, it is not one of the local matters of fact. Therefore, there cannot be a complete circle of explanation because no mosaic is solely responsible for grounding its laws. Laws tell us something more than we can learn by surveying the local matters of the mosaic: they tell us that that is all there is to the mosaic!

In sum, the BSA seems to have the facility, in principle, to fulfill the Humean ideals regarding laws' grounds. But difficulties may arise when we look at particular kinds of laws.

\section{The problem: Idealization laws}

When we come across a complex situation for which a perfectly accurate law is beyond either our knowledge, our ability to solve, or our practical requirements, then we can often idealize the situation. We do this either by abstracting away from the effects of certain causally incidental influences or by fixing variables to some appropriate value (Weisberg 2007; Elgin 2004; Strevens 2008; Potochnik 2017). The result of such idealization is a model (e.g., an equation) that is used to describe, predict, or explain real-world behavior despite not being entirely accurate. For instance, the Newtonian constitutive equation for incompressible fluids is represented as follows:

$$
\tau=-\mu \frac{\partial u}{\partial y}
$$

where $\tau$ is the shear stress of the fluid, $\mu$ is the steady-shear viscosity, and $d u / d y$ is the velocity variation across the $y$-direction transverse to the direction of flow. The equation is a powerful tool for thermodynamic and engineering pedagogy, explanation, prediction, and control; any Humean should want to account for this. Even so, there are no truly incompressible fluids. Real fluids vary in density with changes in pressure. The constitutive equation is used to idealize real fluids. It need not give an entirely accurate description of a fluid-dynamical system but finds applicability in the 
assumption that starting with facts that abstract away from the complexity of the real world is a good way to explain those systems.

There has been a surge of interest in idealization (e.g., Elgin 2004; Weisberg 2013; Potochnik 2017), and it's easy to see why. Idealization is everywhere in science. Many idealizations are characterized by the use of known idealization laws. Examples include Gresham's law and the laws of supply and demand (from economics), Farr's law (from epidemiology), Werner's law (from geology), Wallace's law (from evolutionary biology), the ideal gas law (from thermodynamics), the logistic and Lotka-Volterra equations (from ecology), Snell's law (from optics), and (more controversially) component-force laws (from classical mechanics). But despite their ubiquity, idealizations and their associated laws are not like a traditional philosopher's conception of laws of nature, as (e.g.) strict, true, deterministic, and widely instanced generalizations. In particular, we'll see that idealization laws present a problem for orthodox Humean thinking.

Following on from section 2.2, I take it that equations like equation 1 are not themselves laws but rather specifications of some behavioral characteristic predicable of certain sorts of systems. As we saw, it is reasonable to think that laws that emerge from a best system condition such behavior on some or other system type (as per schema 1). It is just this fact that makes it sensible to talk of laws as representing (or just being, under some ways of talking) regularities in the world. The question, however, is whether idealization laws can be understood in these terms. In the rest of this section, I'll discuss two seemingly exhaustive options for fitting idealization laws into the form of schema 1 . These include "going broad," where the system type is understood to have plenty of instances, and "going narrow," where the system type is understood to have very few, if any. We'll see that there are serious difficulties with both options.

\section{I Going broad}

If we are to fit the idealization law corresponding to equation 1 into the form of schema 1 , one option is to "go broad" and consider the relevant system type to be being a real fluid (RF), providing the following generalization:

$$
(x)\left(\operatorname{RF}(x) \rightarrow \tau(x)=-\mu(x) \frac{\partial u(x)}{\partial y(x)}\right) .
$$

Generalization 1 has plenty of instances (i.e., systems that satisfy the antecedent), but the consequent will falsely predict the behavior of most if not all of them. Following the suggestion of Braddon-Mitchell, the Humean might feel relaxed about this due to the fact that the best deductive system is permitted to be somewhat lossy (see section 2.2). But there is a good reason why it is implausible to assume that the trade-off between (on the one hand) truth and (on the other hand) strength and simplicity can account for the imputed inaccuracy of idealization laws.

Idealization laws invariably correspond with more precise generalizations that condition behavior on just the same broad class of system. For instance, all real fluids in general will be much better approximated by the Newtonian constitutive equation for compressible fluids: 


$$
\tau=-\mu \frac{\partial u}{\partial y}+\left(\frac{2}{3} \mu-\kappa\right)(\nabla \cdot v) I
$$

The additional variables to those in equation 1 are for bulk viscosity $(\kappa)$ and bulk flow velocity $(v)$ ( $I$ is the identity tensor). This more precise behavioral equation has the idealizing equation (equation 1) as a special case. As Morrison points out in her Introduction to Fluid Mechanics (with equations' names substituted for continuity),

for incompressible fluids, $\nabla \cdot v=0$ and equation 2 reduces to equation 1 . $(2013,867)$

The same goes for other cases of idealization. For example, the idealizing RayleighJeans formula for black-body radiation at low frequency can be obtained from the equation in Planck's law by putting the Planck constant to zero; the idealized dynamical behavior of an undampened oscillator can be obtained by putting the viscosity of non-Hookean springs to zero; the ideal gas equation can be obtained from the Van der Waals equation by putting the values for intermolecular force and size to zero; the Malthusian growth equation can be obtained from the Lotka-Volterra equations by setting the predatory population to zero. In each case, the derived equation (often preferred in practice) can be considered a special case of the more complex equation, obtained when appropriate terms are set to a constant (often zero). In each case, the more complex and precise equation is applied in practice to a set of systems that includes that to which the original and simpler equation applies. Indeed, one might reasonably suspect that the existence of such a mathematical relationship with a more accurate equation is the mark of idealization, since idealizing equations are always idealizations from some less idealizing model.

But the correspondence of idealizing equations with more accurate equations implies that the inaccuracy of broad idealizing generalizations like generalization 1 cannot be mitigated by permitted lossiness. That's because there's always another generalization that is more accurate (at some small cost to its simplicity) and that is inconsistent with that broad idealizing generalization. In the fluid dynamical case, for example, we have the following generalization:

$$
(x)\left(\operatorname{RF}(x) \rightarrow \tau(x)=-\mu(x) \frac{\partial u(x)}{\partial y(x)}+\left(\frac{2}{3} \mu(x)-\kappa(x)\right)(\nabla \cdot v(x)) I\right) \text {. (generalization 2) }
$$

Generalization 2 is clearly a law of fluid dynamics if generalization 1 is. From the Humean perspective, it is more accurate for a broader range of real fluids at a presumably justifiable increase in complexity. From the perspective of scientific practice, equation 2 is employed widely in fluid dynamics, and we would certainly not want to preclude its involvement in a law for the sake of including the employment of the less accurate equation. However, the two broad generalizations, generalization 1 and generalization 2, are inconsistent. At most, one can be a theorem of the best system for fluid dynamics. The strategy of "going broad" therefore seems to force us to choose between candidate laws involving equations, both of which we would like to feature in the laws of fluid dynamics.

In sum, we'd better find some other way of rendering idealization laws like that for constitutive flow because the strategy of "going broad," as we're currently conceiving 
it, brings the candidate idealization laws into conflict with those further candidate laws constructed in the same schematic way using more accurate equations of which the idealizing equation is a special case. The obvious response to neutralize the conflict is to narrow the scope of one of the generalizations, and if either candidate is to have its scope narrowed, it is surely not that of the more accurate law, since it is better at predicting the behavior of that broad class of systems. ${ }^{3}$

\subsection{Going narrow}

Because we've tried "going broad" with idealization laws' system types, the alternative seems to be to "go narrow" and consider the relevant system type of whatever idealization law corresponds with equation 1 to be more tailor-made for the particular behavioral function. The obvious choice for our central example is being an incompressible Newtonian fluid (IF), as in the following generalization:

$$
(x)\left(\operatorname{IF}(x) \rightarrow \tau(x)=-\mu(x) \frac{\partial u(x)}{\partial y(x)}\right) .
$$

At the very least, such a formulation has the merit of fitting our common parlance of the law as one "for incompressible fluids." In practice, an incompressible fluid is any fluid with a Mach number of less than 0.3, implying a change in density of less than $5 \%$. If IF is interpreted this way, then it will have a narrower class of instances than RF, although it will not be completely lacking in them. Nevertheless, it remains implausible for the Humean to interpret the idealization law for constitutive incompressible flow in this way. Unless we also interpret RF as being satisfied only by fluids with a Mach number of greater than or equal to 0.3 , then generalization 3 and generalization 2 will also be inconsistent because some of their instances will be shared while the predictions will be different. However, interpreting generalization 2 as concerning all fluids is so much simpler than bifurcating the class of real fluids into a subclass described by equation 2 and a subclass described by equation 1 (I assume there must be a law covering the compressible fluids). Moreover, equation 2 is also more accurate than equation 1 , not just for real fluids with a Mach number of $>0.3$ but also for those with a Mach number of $\leq 0.3$. Consequently, generalization 3 will not be counted as a law if its instances include just any fluid with a change in density of less than $5 \%$.

This kind of reasoning shows in general that the only way to narrow the scope of an idealization law's antecedent condition that has a chance of avoiding conflict with more accurate counterparts is to narrow it to that class of systems for which the more accurate counterpart provides no predictive improvement. In the fluid dynamical case, this would involve treating IF as satisfied by only truly incompressible fluids (i.e., those whose density does not change under pressure). In that case, of course, generalization 3 turns out to be vacuous (and similarly for other idealization laws).

Although it is not incumbent on a Humean who adopts the BSA to ground every law in the regularity it describes (i.e., not every law needs to be grounded in its own instances), it is fairly disastrous to think that the constitutive law for incompressible

\footnotetext{
${ }^{3}$ of course, it could well be that neither is satisfactory in its broad form if there is a third even more accurate equation applying to at least the same class of systems.
} 
flow-or indeed any idealization-is one of these. There are at least two reasons for this.

First, the issue of generalization 3's vacuity is not just that there happen to be no instances but also that instances are impossible according to the laws of fluid dynamics. After all, there are laws of fluid dynamics (e.g., the total force law) that entail that all fluids are somewhat compressible under pressure. Hence, it is a consequence of the laws themselves that nothing satisfies the antecedent of generalization 3 . This makes generalization 3 a trivial theorem of the best system because any generalization with that antecedent condition would be a theorem. ${ }^{4}$ Even if the Humean is willing to stomach labeling such theorems "laws," there is surely a difference between those of value in scientific practice (e.g., idealization laws and nontrivial axiomatic laws) and trivial theorems like, "If something is an incompressible fluid, then anything goes." Yet Humeans seem to commit to a rejection of this distinction by narrowing the scope of the system type in this way.

Note that this issue of triviality does not apply only to the fluid-dynamical case. Presumably, it is a law of ecology that populations always have some external pressures (predation, food sources, environmental hazards, other evolutionary pressures, etc.). This entails not only that there isn't any system that exactly obeys the Malthusian exponential growth equation but also that there couldn't be. ${ }^{5}$ Hence, any attempt to narrow the system type to which the Malthusian growth equation applies - so that it comes out true and consistent with more accurate ecological laws with a broader scope-will be trivially true. Malthusian growth will "apply", but so will any functional behavior because the antecedent is unsatisfiable. I take it that similar reasoning will hold for other idealization laws (e.g., the ideal gas law, law of supply and demand, etc.).

The risk of triviality is problem enough for the "going narrow" approach. But a further and perhaps even less avoidable problem is that going narrow makes it difficult to see how idealizing equations can have the explanatory relevance they have to real-world situations. The explanatory importance of idealization laws is nicely brought out by Elgin in a discussion of Snell's law, an idealization of the contributory influence of perfect media boundaries to actual (often anisotropic) refraction phenomena.

Sometimes it is useful to first represent a light ray as conforming to Snell's law, and later introduce 'corrections' to accommodate anisotropic media.. .. [I]f we are interested in optical refraction in general, it might make sense to start with a prototypical case, and then show how anisotropy perturbs. By portraying anisotropic cases as perturbations, we point up affinities that direct comparisons [e.g., between the paths of two actual light rays] would not reveal.. .. Showing how a variety of cases diverge from the prototypical case contributes valuable insights into the phenomenon we are interested in. And what makes the case

\footnotetext{
${ }^{4}$ Thanks to an anonymous referee for stressing this point to me.

${ }^{5}$ There is also reason to think this is due to the intrinsic character of the equation because it predicts that populations change along a continuum of values (McLoone 2019). Any more accurate law that has the Malthusian laws as a special case will therefore share in this particular issue too.
} 
prototypical is not that it usually obtains, but that it cleanly exemplifies the features we deem important. (2004, 117-18)

Elgin's example draws attention to the thought that idealization involves abstraction from real-world cases in order to explain those very real-world behaviors. By idealizing, we draw attention to the affinity that many real-world cases have, which we could not do if we only used more accurate but also more complex laws. Idealization laws, it seems, are explanatorily relevant to real-world systems.

Idealization laws also furnish an important theoretical basis for more complex laws. This point is made by Woody with reference to the ideal gas law:

Descriptions surrounding application of the law, in textbooks and classrooms, suggest instead that idealization underwrites both its broad application and its explanatory status.. .. This vision [of an ideal gas] guides model construction for actual gases, and when the law poorly approximates actual gas behavior, properties are conceptualized as deviations from the ideal. In this way, the law's mathematical structure provides inferential scaffolding for the treatment of all gases, not only those for which it is a good approximation. Thus, the ideal gas law's role in practice is not essentially descriptive, but rather prescriptive; by providing selective attention to, and simplified treatment of, certain gas properties (and their relations) and ignoring other aspects of actual gas phenomena, the ideal gas law effectively instructs chemists in how to think about gases as they are characterized within chemistry. $(2015,4)$

Here, Woody is drawing attention to the ideal gas law's "cognitive role in chemistry" in helping chemists to better understand the behavior of real gases. The point is that the idealization laws aid our reasoning and understanding in a way that shows them to be explanatorily relevant-in part, perhaps, by setting conceptual constraints-to what more accurate laws must be like. Notice that this is the case even if, in practice, it is known that the idealizing equations are only predictively accurate in a small number of cases (if any). Idealizations have explanatory relevance even in cases when they are poor predictors.

The observation of idealizing equations' wide application and explanatory relevance to real-world situations heavily undermines the thought, implied by the "going narrow" approach, that the laws in which they are involved concern antecedent system types that aren't satisfied by anything in the world (or only extreme cases). By modifying the antecedent in this way, we risk making idealization laws irrelevant (cf. Cartwright 1983, 57-58). Yet idealization laws, it seems, are about real, nonideal cases. $^{6}$

In sum, the "going narrow" strategy of rendering idealization laws' antecedent system type is at least as undesirable as the "going broad" strategy appears to be. This is because there's no way to narrow the scope of the system type in a way that removes the risk of conflict with more accurate candidate laws but avoids rendering them vacuous (or applying to only some very extreme class of cases). The existence of

\footnotetext{
${ }^{6} \mathrm{~A}$ non-Humean way of putting this would be to say that the law for incompressible flow governs compressible fluids.
} 
vacuous laws might not be an issue for the Humean per se, but in the particular case of the attribution of idealizing behavior it risks (i) the resulting generalization being a trivial theorem of the relevant deductive system (because there may be laws that preclude the existence of instances of the system type), and (ii) the resulting generalization failing to have a system type appropriate for the kinds of practical explanatory applications to which the idealizing equations in fact get applied.

\subsection{A contrast in problems}

The problem raised for idealization laws is essentially a dilemma of logical form. We want to find a way to identify a system type about which the law can be said to concern that renders the law nontrivial and nonvacuous. But we need to find a way to do so that doesn't conflict with the fact that there is clearly a more accurate law that covers at least the same systems that the idealization law covers. If we choose the same broad system type as the more accurate law, then there is inconsistency in the deductive system, and the more accurate law is clearly the preferable theorem to maintain. If we choose a narrower system type, then there is hopeless triviality and a loss of explanatory power.

Something like this problem has been observed in a more general assessment of Humean approaches to special science laws by Backmann and Reutlinger (2014), who identify both "Lange's dilemma" (after Lange 1993), between laws' triviality and falsity, and "Cartwright's dilemma" (after Cartwright 1983), between laws' vacuity and falsity. Both dilemmas, they claim, undermine the possibility of the BSA accommodating special science laws that are invariably idealizing or require ceteris paribus clauses. They argue, in particular, that the problems remain even when granting the modifications proposed by Cohen and Callender (2009), who explicitly suggest that their science-relativization may help avoid such troubles. ${ }^{7}$

I'm focusing on idealization laws here, thereby leaving so-called "ceteris paribus laws," which have some or other explicit modifier (cf. Fodor 1974; Pietroski and Rey $1995)$ to the system type, to one side. Consequently, some of the issues raised by Backmann and Reutlinger (2014) do not apply. In particular, the triviality complaint raised in the foregoing is not quite that exposed by Lange's dilemma. Lange was concerned that ceteris paribus clauses could be used to trivialize a law by building into the antecedent the behavior mentioned in the consequent, or else by allowing for any kind of behavior to be consistent with the generalization. The triviality worry raised in the present discussion, however, is rather that the laws might entail that there are no instances of an idealization law's system type, and so whatever behavior it attributes to the system type will be trivially satisfied. Nevertheless, an answer to the problem of idealization laws' logical form will surely go some way toward addressing the concerns raised by them.

\footnotetext{
${ }^{7}$ Backmann and Reutlinger grant that the admission of lossy laws may have more luck. I certainly think such an admission is necessary, but the foregoing discussion makes clear that it is also insufficient, given the existence of more accurate laws for the same system type.
} 


\section{The solution: "Going meta"}

Going broad and going narrow are only exhaustive options if one assumes that idealization laws must maintain the form of schema 1. The way out of the dilemma just posed for idealization laws is, therefore, to break with this assumption. Evidently, if schema 1 is a mark of laws among the theorems of a best system, as I think it is, then this way out will require us to think of idealization laws as something other than the laws of a best system. What I propose is that we think of them as meta-lawsspecifically, theorems of a "meta-ized best system" with a certain schematic form. In section 4.1, I give the details of this "going meta" solution. In section 4.2, I say why it improves on the foregoing approaches to idealization laws. In section 4.3, I say why it fits the Humean credentials in a satisfying way.

\section{I Idealization laws as meta-laws about super laws}

Following Cartwright (1983, 70), let's refer to those more accurate generalizations concerning the same broad class of systems to which idealizing equations also seem relevant as the idealization's corresponding super law. The original idea behind the notion of a super law is that such laws "take into account all the relevant interactions into which a system can enter" (Corry 2009, 166), although I will suggest later that this is something the Humean should probably not want to commit to. Such laws therefore diverge from idealization laws that only take account of some subset of interactions, like when equation 1 fails to include variables for bulk viscosity and bulk flow velocity on shear stress. Therefore, under this interpretation, the behavioral functions in super laws are superlative with respect to their ability to accurately predict the behavior of physical systems, at least in comparison to any other function involved in the deductive system for the relevant domain (e.g., fluid mechanics).

Crucially, idealizing equations are related to equations featuring in super laws by being special cases of them, that is, by being instances of the super laws' equations that result from substituting some variables for constants (which may, e.g., be finite, complex, zero, or a limit). In principle, then, there might be a sequence of idealizing equations related by virtue of each in the sequence having some variable that is a constant in the preceding equations until finally reaching that full set of variables employed in a super law. Of course, it might happen that we don't precisely know a super law corresponding to some idealization equation. Nevertheless, if we know a law is an idealization, then we know that some super law is out there-or at least, that's the idea.

Despite being an initial cause for concern, the existence of super laws can in fact be used by Humeans to construct a plausible view of idealization laws. Here's a first pass. Because any idealizing equation $E$ itself is a special case of some corresponding super law's behavioral function, we can attribute to the same system type $S$ to which the super law applies the property of being such that its instance's behaviors are described by a behavioral function that has $E$ as a special case. It's a good start, but it's not going to be quite what we need because it is trivially satisfied. Any equation is a special case of some behavioral function that accurately describes some finite class of systems. What more is needed, I take it, is the observation that the behavioral function that has $E$ as a special case is that of a (super) law. That is, for any idealizing equation $E$ explanatorily relevant to some system type $S$, the following sort of statement is true: 
There is a law $(\mathrm{x})(\mathrm{S}(\mathrm{x}) \rightarrow \mathcal{F}(\mathrm{x}))$, suchthat $\mathcal{F}$ hasEasaspecialcase.

(schema 2)

It is this form I propose the Humean understands idealization laws to have. So, for example, in the case of the Newtonian constitutive equation for incompressible flow, the corresponding idealization law looks as follows:

There is a law $(x)(R F(x) \rightarrow \mathcal{F}(x))$, such that $\mathcal{F}$ has

$$
\tau(x)=-\mu(x) \frac{\partial u(x)}{\partial y(x)} \text { as a special case. }
$$

(generalization 4)

Two things are of note about instances of schema 2 (like generalization 4). First, they do not have the form of a generalized conditional. The quantifier that takes the widest scope is an existential quantifier, not a universal quantifier. As a consequence, they do not have the form of schema 1. However, they do imply that there must be laws of that form. Hence, second, instances of this schema make reference to laws and so are, in some reasonable sense, "meta-laws." Both characteristics imply that idealization laws cannot be laws in the standard way Humeans have understood them. Strictly speaking, laws under the BSA are theorems of the best deductive system that are generalizations (Lewis 1973, 73), arguably of the form schema 1. This means that even if idealization laws were to feature in a best system, they couldn't be laws. However, the deductive system takes as input only facts about the mosaic, and because the Humean eschews a metaphysically realist view of laws, no such facts will be facts about what the laws are. Hence, if idealization laws are to have the form schema 2, they can't even be in a best system.

If idealization laws aren't in a best system then where do they come from? Key to the proposal is the idea that idealization laws can be accommodated by the Humean, not as a theorem of a best system's axioms but as a theorem of a different yet related set of axioms. Take generalization 4. Neither generalization 1 nor generalization 2 is logically sufficient to entail it. But the statement that either generalization is a theorem of a best system (and hence a law) would be. For example, if-as seems the more likely of the two-generalization 2 were a theorem of a best system, then the statement that it is such a theorem would entail (under Humean best systems theory) that generalization 2 is a law. And given that generalization 2 entails that all real fluids' shear stress $(\tau)$, steady-shear viscosity $(\mu)$, and velocity variation $(d u / d y)$ are mutually predictable according to some function that has equation 1 as a special case, then the statement that generalization 2 is a law will entail generalization 4.

In general, then, although Humeans will want to say that the (first-order) laws for some domain of properties are the expressions of the form of schema 1 that are theorems of the axioms of a best systematization of the distribution of those properties, they should say something different for the idealization laws for that domain. Under the "going meta" proposal, idealization laws are expressions of the form of schema 2, which are theorems of a set of axiomatic statements that state, for each axiom $L$ of the best system, that $L$ is an axiom of the best system. ${ }^{8}$ Call this alternative deductive system the

\footnotetext{
${ }^{8}$ of course, for the entailment to go through, the theorem must be referred to in a way that makes its generalization transparent, as in schema 2. It wouldn't do, for instance, to name the theorems and then refer to the theorems by their names.
} 
"meta-ized best system". ${ }^{9}$ Notice that it will follow deductively from all the axioms of this meta-ized system that all the theorems of the corresponding best system are laws, too, and that all the special cases of all the behavioral functions attributed to certain system types by theorems of the best system are special cases of functions attributed by laws about those system types. There will, therefore, be instances of schema 2 in a meta-ized best system if there are laws in the its corresponding best system. According to the current proposal, all of these are idealization laws.

\subsection{Why going meta is better}

Like the "going broad" proposal considered earlier, the "going meta" proposal keeps the relevant system type (whose instances' behavior the law ultimately concerns) broad. For example, the relevant system type for the idealization law for constitutive incompressible flow concerns all real fluids. Similarly, the ideal gas law will concern all gases, the Malthusian growth equation all populations, and so on. In doing so, the proposal avoids the troubles associated with narrowing the law's system type. As we learned in section 3.2, going narrow seems doomed to render idealization laws disastrously trivial and explanatorily vacuous, neither of which is a plausible characteristic of idealization laws. Yet the problem with keeping the system type broad, or so it seemsed, is that idealization laws then come into conflict with their super laws. However, the conflict with super laws only occurs when it is assumed that the idealization laws and their super laws will have the same logical form (viz. that of schema 1). The "going meta" proposal gives up this assumption. Idealization laws do not, after all, have the same schematic form as their super laws. Rather, they have the form of meta-laws (specifically, schema 2). As such, they are no longer inconsistent with their super laws. Indeed, they are partly grounded in them! Idealization laws are theorems derived from the statement (ultimately from axioms in the meta-ized best system) that their super laws are theorems of a best system.

So, far from being inconsistent with their super laws, the "going meta" proposal actually makes idealization laws reliant on them. But can the Humean rely on the existence of the required super laws? One might have reason for skepticism. Earlier, the Newtonian law for compressible flow (generalization 2) was presented (implicitly) as a candidate super law for the idealization of incompressible flow. As it happens, however, the constitutive equation for compressible flow involved in that law does not itself describe any fluid perfectly accurately because no fluid is precisely Newtonian (i.e., has a viscous stress linearly proportional to the strain rate), and some fluids diverge considerably (e.g., peanut butter and mayonnaise). This might lead to the thought that generalization 2 cannot, after all, be a corresponding super law.

But that is not necessarily the right inference. Recall that the Humean who takes truth to be a desideratum in choosing the best system for some domain can permit some lossiness in the system so long as it scores well on strength and simplicity. The reason the lossiness of the initial candidate idealizing generalization (generalization 1) went unmitigated was because (a) it was very lossy in some cases,

\footnotetext{
${ }^{9}$ Not to be confused with a meta-best system, which best systematizes the theorems of the best system according to desiderata of (e.g.) simplicity and strength (cf. Lange 2007, 479). The meta-ized system is not a systematization of anything. It is a system whose axioms are one-to-one correspondent with a best system's axioms.
} 
and (b) we could name generalization 2 as a rival candidate generalization that would come into conflict with it. It's not obvious that generalization 2 is problematic to the same extent. Regardless, what is crucial is that whatever our verdict about the status of generalization 2, there is some generalization of the form schema 1 that appears in the best system for fluid dynamics that (a) is accurate enough such that any loss is mitigated by the overall strength and simplicity of the system to which it contributes, and (b) there is no further generalization in that same system that concerns the same system type and attributes a functional behavior that has the first generalization's attributed behavior as a special case.

\subsection{Satisfying Humean considerations (again)}

What's already been presented is enough, I think, to show that idealization laws have the right structure to avoid the dilemma raised in section 3 . The solution is to reject the idea that idealization laws are laws, in the typical Humean sense of being theorems of a best system. But it would be far from adequate to take the position that the Humean requirements on laws do not apply for that reason. Idealization laws aren't pure imaginative constructs and must, for the Humean, be ultimately grounded in a mosaic. Idealization laws are also clearly empirically accessible-indeed, arguably more so than their super laws. Finally, as we have seen in section 3.2, idealization laws are explanatorily important. So if the proposal for Humean idealization laws is to be at all plausible, it must at least cohere with the same three requirements for laws. Thankfully, it does.

Idealization laws have often been posed as problems for the Humean on account of their lack of metaphysically sufficient grounds within the Humean mosaic (Cartwright 2009; Corry 2009; Cartwright 2017; Backmann and Reutlinger 2014). But there is no special problem of metaphysical grounds under the current view because idealization laws are grounded ultimately in some or other mosaic, along with totality facts. That's because a mosaic, plus a totality fact about the extent of the mosaic, grounds a best system's generalizations, and the best system's generalizations, plus the totality fact about the availability of other systems, in turn ground a corresponding meta-ized best system, of which idealization laws are theorems. As before, I take it that the commitment to totality facts provides no unique problem for the Humean.

The approach of understanding idealization laws as meta-laws might seem, at a glance, to render idealization laws too far removed from the goings-on of the mosaic to satisfy the requirement of empirical accessibility. But I think we can assuage much of this kind of concern. Note that it is not incumbent on Humeans to argue that the super laws that help ground idealization laws must be epistemically prior to or more accessible than their corresponding idealization laws. It is therefore not straightforwardly a problem that super laws are often only discovered much later than the derived idealization laws, if at all. Humeans can also provide a plausible story about why the idealization laws would be discovered sooner than, and even used to predict, their super laws. Idealizations' mathematical simplicity and approximation to realworld phenomena (when other factors are minimal) make them obvious inferences from experimental data. This can happen either as a conscious effort to idealize or as a result of discrepancies being falsely attributed to experimental error. The merit of 
the "going meta" approach is that it shows why the idealization laws are legitimate inferences from experiments on real-world systems.

Lastly, we come to the requirement that the "going meta" proposal should be able to show why idealization laws have the explanatory relevance they have. As already remarked on, under this proposal, idealization laws get to keep their breadth without coming into conflict with other laws. For example, the idealization law for constitutive incompressible flow (generalization 4) makes a claim about all real fluids, and so it makes sense of the evident fact that equations like equation 1 are explanatorily relevant to real-world systems. Insofar as they do this, idealization laws are able to explain the goings-on of the mosaic in much the way that first-order laws are. Furthermore, by rendering idealization laws in the form of schema 2, idealization laws gain an extra dose of explanatory power. By attributing to the broad class of systems a behavior that has some explicit equation as a special case, idealization laws are able to draw specific attention to a subset of (perhaps particularly important) causal relationships around which more complex laws are built. This all seems to support the increased pedagogical and cognitive explanatory power of idealization laws emphasized by Elgin and Woody in the above quotations.

of course, according to the proposal, the kind of explanatory relevance idealization laws have to their more complex super laws is not one of ontological priority. For the Humean who adopts this meta-conception of idealization laws, idealization laws are parasitic on their super laws. Consequently, the conception will not do justice to the intuition of, for example, Cartwright, who has long argued that "what unified [super] laws dictate should happen, happens because of the combined action of laws from separate domains" (1983, 70).

It's not my aim to show that idealization laws under the proposed Humean conception satisfy that intuition. The Humean should not feel compelled to do justice to the thought that super laws are the way they are because idealization laws are as they are, at least not in the same way Humeans are (perhaps) compelled to make sense of the thought that the mosaic is the way it is because of the laws.

As we saw earlier, for the Humean, first-order laws explain the mosaic because they are part of a unifying systematization of it. Idealization laws should inherit some of that explanatory power over the mosaic too. But granting that the form of explanation is unification, there does not seem to be any reason, from a Humean perspective, to think that idealization laws unify their super laws in any way (if anything, it's the other way around: the super laws will unify a number of idealization laws formed from different fixings of variables). However, if the charge is that the Humean should be seeking to make sense of a more weighty kind of explanatory priority for idealization laws, then the Humean can simply respond that such a demand falls into the bracket of expectations they eschew in general (e.g., that laws govern, that they are productive, etc.).

Moreover, the Humean can reject this demand without having to give up making sense of what is perhaps driving the Cartwrightian intuition: that idealization laws concern core causal features of systems that are subject to further interference in situ (Weisberg 2007; Corry 2009; Cartwright 2017). Because of the posited relationship of idealizing equations being special cases of super laws' equations, any causal components described in an idealizing equation for some physical system will be causal components also described in a super law's equation for that system. The 
Humean simply leaves it up to context-variable scientific interests to say what counts as a "core causal component" and what counts as "interference."

A different kind of worry related to idealization laws' explanatory power concerns their modal robustness. Under a traditional Humean view of nomological possibility, the content of a law is nomologically necessary, but it is not nomologically necessary that such content is that of a law. ${ }^{10}$ Consequently, claims to the effect that such and such axiom of a best system is a law will not be as modally robust as the axiom itself. The upshot for the "going meta" proposal is that idealization laws will be modally weaker than the super laws from which they are derived.

How much of a problem is this? Perhaps not so much. Notice that we can still admit that whenever the super law is a law, so is the idealization law. Moreover, in worlds where a super law of our world is true but is not a law, its corresponding idealization laws will still employ equations that idealize the behavior of the systems that the super law does. Nevertheless, because of their weakened modal status, the proposal technically renders instances of schema 2-and so idealization laws-accidental regularities, which can seem, at the very least, highly counterintuitive and potentially also inconsistent with their explanatory relevance.

I think the best response the Humean can make to this issue is one they should probably feel compelled to make anyway, and that is to assert a condition of nomic preservation (Roberts 2008; Lange 2009). Notice that it is counterintuitive to say that if I were to have done differently, the laws might have been different. But that's something the Humean must accept when following traditional lines. Similarly, it has been widely recognized that the counterfactuals of sparse worlds (e.g., containing a single electron) under such a view are liable to be counterintuitive too (Carroll 1994). The Humean is not committed to the traditional line. An alternative is to say that laws remain laws under counterfactual supposition up to inconsistency with the counterfactual conditional's antecedent (see, e.g., Roberts 2008, 191). That means, roughly, that what it is for a statement of the form "it is a law that $p$ in $w$ " to be true is not a matter of whether $p$ is an axiom of a best systematization of some mosaic in $w$ but whether it is true at $w$ and an axiom of a best systematization of some mosaic in the world of utterance. ${ }^{11}$ As a consequence of this, laws will remain laws under consistent counterfactual supposition. Drawing the analogous move with corresponding meta-ized best systems, the Humean will assert that idealization laws remain idealization laws under counterfactual supposition up to inconsistency with the counterfactual conditional's antecedent. This ensures that idealization laws' modal robustness is more equal to that of their super laws, as well as making sense of a number of other issues already facing Humean thinking about laws.

In sum, the proposal to take idealization laws as meta-laws about super laws seems to fulfill its duty of satisfying the Humean basic requirements described in section 2.1 while also capturing the more demanding explanatory intuitions motivated in section 3.2. I considered a couple of objections to this proposal but found neither to be a significant stumbling block.

\footnotetext{
${ }^{10}$ Thanks to an anonymous reviewer for raising this issue.

${ }^{11}$ The details will in fact need to be a little more complicated; see Roberts (2008, chaps. 5-6). A further alternative is for the Humean to adopt a notion of "nomothetic explanation" to contrast with that of "metaphysical explanation" (Bhogal, forthcoming).
} 


\section{Conclusion}

If one has Humean inclinations, what account should one provide for idealization laws? It is tempting to find a place for them in a best system, but as I've demonstrated, this is far from easy. Either idealization laws will come into conflict with the super laws that attribute more accurate behavior to a wide class of real-world systems, or they will be trivial and explanatorily vacuous on account of not attributing behavior to any system. My suggestion has been to take idealization laws not to be theorems of a best system and to consider them as theorems of a specific schematic form of a meta-ized best system that has an axiom corresponding to each axiom $L$ of a specific best system that states that $L$ is an axiom of a best system. By doing this, we retain idealization laws' relevance to the real world without coming into conflict with the super laws from which they idealize.

Acknowledgments. This article is what emerged from a presentation at the 2016 Society for Metaphysics of Science Conference in Geneva. I would like to thank the audience there for their participation and particularly Helen Beebee for providing commentary. Thanks also go to Mike Townsen Hicks for comments and discussion on the draft that followed. Finally, I would also like to take the opportunity to sincerely thank my two reviewers. The reviewing process is too often needlessly painful for authors, but sometimes reviewers help to significantly improve an author's understanding of their own work and improve the article as a consequence. My experience with this article was undoubtedly of the latter sort. Any success this article has owes much to the reviewers. Any failings are, of course, entirely my own. Much of the work in producing this final version has been funded by the European Research Council (ERC) under the European Union's (EU) Horizon 2020 research and innovation programme, grant agreement No 771509 ('MetaScience').

\section{References}

Armstrong, David Malet. 1983. What Is a Law of Nature? Cambridge: Cambridge University Press.

Backmann, Marius, and Alexander Reutlinger. 2014. "Better Best Systems-Too Good to Be True." Dialectica 68 (3):375-90.

Bhogal, Harjit. Forthcoming. Nomothetic Explanation and Humeanism about Laws of Nature. Oxford Studies in Metaphysics. Oxford: Oxford University Press.

Braddon-Mitchell, David. 2001. "Lossy Laws." Noûs 35 (2):260-77.

Callender, Craig, and Jonathan Cohen. 2010. "Special Sciences, Conspiracy and the Better Best System Account of Lawhood.” Erkenntnis 73 (3):427-47.

Carroll, John. 1994. Laws of Nature. Cambridge: Cambridge University Press.

Cartwright, Nancy. 1983. How the Laws of Physics Lie. Oxford: Oxford University Press.

Cartwright, Nancy. 2009. "Causal Laws, Policy Predictions, and the Need for Genuine Powers." In Dispositions and Causes, edited by Toby Handfield, 6-30. Oxford: Clarendon Press.

Cartwright, Nancy. 2017. “Causal Powers: Why Humeans Can't Even Be Instrumentalists.” In Causal Powers, edited by Jonathan Jacobs, 9-23. Oxford: Oxford University Press.

Cohen, Jonathan, and Craig Callender. 2009. “A Better Best Systems Account of Lawhood.” Philosophical Studies 145 (1):1-34.

Corry, Richard. 2009. "How Is Scientific Analysis Possible?" In Dispositions and Causes, edited by Toby Handfield, 158-88. Oxford: Clarendon Press.

Demarest, Heather. 2017. "Powerful Properties, Powerless Laws." In Causal Powers, edited by Jonathan Jacobs, 38-53. Oxford: Oxford University Press.

Dorst, Chris. 2019. "Towards a Best Predictive System Account of Laws of Nature." British Journal of Philosophy of Science 70 (3):877-900.

Earman, John. 1986. A Primer on Determinism. Dordrecht: D. Reidel.

Elgin, Catherine. 2004. “True Enough.” Philosophical Issues 14 (1):113-31. 
Fenton-Glynn, Luke. 2016. “Ceteris Paribus Laws and Minutis Rectis Laws.” Philosophy and Phenomenological Research 93 (2):274-305.

Fodor, Jerry. 1974. "Special Sciences (Or: The Disunity of Science as a Working Hypothesis)." Synthese 28 (2):97-115.

Friend, Toby. 2016. “Laws Are Conditionals." European Journal for Philosophy of Science 6 (1):123-44.

Friend, Toby. 2022. "The Humean pragmatic turn and the case for revisionary best systems accounts." European Journal for Philosophy of Science 12:1-26.

Hicks, Michael Townsen. 2018. "Dynamic Humeanism." British Journal of Philosophy of Science 69 (4): 983-1007.

Hicks, Michael Townsen. 2021. "Breaking the Explanatory Circle." Philosophical Studies 178 (2):533-57.

Hicks, Michael Townsen, and Peter van Elswyk. 2015. "Humean Laws and Circular Explanation." Philosophical Studies 172 (2):433-43.

Hüttemann, Andreas. 2021. A Minimal Metaphysics for Scientific Practice. Cambridge: Cambridge University Press.

Jaag, Siegfried, and Christian Loew. 2020. "Making Best Systems Best for Us.” Synthese 197 (6):2525-50.

Kimpton-Nye, Samuel. 2017. "Humean Laws in an UnHumean World." Journal of the American Philosophical Association 3 (2):129-47.

Kimpton-Nye, Samuel. 2021a. "Laws of Nature: Necessary and Contingent." The Philosphical Quarterly, pqab062. https://doi.org/10.1093/pq/pqab06244.

Kimpton-Nye, Samuel. 2021b. "Reconsidering the Dispositional Essentialist Canon." Philosophical Studies 178 (10):3421-41.

Lange, Marc. 1993. "Natural Laws and the Problem of Provisos." Erkenntnis 38 (2):233-48.

Lange, Marc. 2007. "Laws and Meta-Laws of Nature: Conservation Laws and Symmetries." Studies in History and Philosophy of Modern Physics 38 (3):457-81.

Lange, Marc. 2009. Laws and Lawmakers: Science, Metaphysics, and the Laws of Nature. Oxford: Oxford University Press.

Lange, Marc. 2013. "Grounding, Scientific Explanation, and Humean Laws." Philosophical Studies $164(1): 255-61$.

Lange, Marc. 2018. "Transitivity, Self-Explanation, and the Explanatory Circularity Argument against Humean Accounts of Natural Law." Synthese 195 (3):1337-53.

Lewis, David. 1973. Counterfactuals. Oxford: Blackwell.

Lewis, David. 1983. "New Work for a Theory of Universals." Australian Journal of Philosophy 61 (4):343-77.

Lewis, David. 1986. Philosophical Papers II. Oxford: Oxford University Press.

Lewis, David. 1994. “Humean Supervenience Debugged.” Mind 103 (412):473-90.

Loewer, Barry. 1996. "Humean Supervenience." Philosophical Topics 24 (1):101-27.

Loewer, Barry. 2012. “Two Accounts of Laws and Time." Philosophical Studies 160 (1):115-37.

Marshall, Dan. 2015. "Humean Laws and Explanation." Philosophical Studies 172 (12):3145-65.

Maudlin, Tim. 2007. The Metaphysics within Physics. New York: Oxford University Press.

McLoone, Brian. 2019. "Thumper the Infinitesimal Rabbit: A Fictionalist Perspective on Some 'Unimaginable' Model Systems in Biology." Philosophy of Science 86 (4):662-72.

Miller, Elizabeth. 2015. “Humean Scientific Explanation." Philosophical Studies 172 (5):1311-32.

Morrison, Faith. 2013. An Introduction to Fluid Mechanics. Cambridge: Cambridge University Press.

Pietroski, Paul, and Georges Rey. 1995. "When Other Things Aren't Equal: Saving Ceteris Paribus Laws from Vacuity." British Journal of Philosophy of Science 46 (1):81-110.

Potochnik, Angela. 2017. Idealization and the Aims of Science. Chicago: University of Chicago Press.

Roberts, John T. 2008. The Law-Governed Universe. Oxford: Oxford University Press.

Schrenk, Markus. 2007. The Metaphysics of Ceteris Paribus Laws. Frankfurt: Ontos.

Schrenk, Markus. 2014. "Better Best Systems and the Issue of CP-Laws." Erkenntnis 79 (10):1787-99.

Schrenk, Markus. 2017. "The Emergence of Better Best System Laws." Journal for General Philosophy of Science 48 (3):469-83.

Shumener, E. 2021. "Humeans Are Out of This World.” Synthese 198 (6):5897-916.

Strevens, Michael. 2008. Depth: An Account of Scientific Explanation. Cambridge, MA: Harvard University Press. 
Tahko, Tuomas E. 2015. "The Modal Status of Laws: In Defence of a Hybrid View.” The Philosophical Quarterly 65 (260):509-28.

Weisberg, Michael. 2007. “Three Kinds of Idealization.” Journal of Philosophy 104 (12):639-59.

Weisberg, Michael. 2013. Simulation and Similarity: Using Models to Understand the World. Oxford: Oxford University Press.

Woodward, James. 2014. "Simplicity in the Best Systems Account of Laws of Nature." British Journal of Philosophy of Science 65 (1):91-123.

Woody, Andrea. 2015. "Re-Orienting Discussions of Scientic Explanation: A Functional Perspective." Studies in History and Philosophy of Science 52:79-87.

Cite this article: Friend, Toby. 2023. "How to Be Humean about Idealization Laws." Philosophy of Science 90 (1):150-170. https://doi.org/10.1017/psa.2022.12 